MATHEMATICS OF COMPUTATION

Volume 75, Number 254, Pages 997-1013

S 0025-5718(05)01811-9

Article electronically published on November 29, 2005

\title{
CM-FIELDS WITH RELATIVE CLASS NUMBER ONE
}

\author{
GEON-NO LEE AND SOUN-HI KWON
}

\begin{abstract}
We will show that the normal CM-fields with relative class number one are of degrees $\leq 216$. Moreover, if we assume the Generalized Riemann Hypothesis, then the normal CM-fields with relative class number one are of degrees $\leq 96$, and the CM-fields with class number one are of degrees $\leq 104$. By many authors all normal CM-fields of degrees $\leq 96$ with class number one are known except for the possible fields of degree 64 or 96 . Consequently the class number one problem for normal CM-fields is solved under the Generalized Riemann Hypothesis except for these two cases.
\end{abstract}

\section{INTRODUCTION}

In O1], Odlyzko proved that there are only finitely many normal CM-fields of a given class number (see also $[\mathrm{S}]$ ). In [H], Hoffstein showed unconditionally that normal CM-fields with relative class number one are of degrees $\leq 434$ and determined upper bounds for their root discriminants. Recently, Bessassi $([\mathrm{B}])$ improved Hoffstein's bounds: he showed that normal CM-fields with relative class number one are of degrees $\leq 266$ and that if the Generalized Riemann Hypothesis is true, then they are of degrees $\leq 164$. Usually, to solve the class number one problem for normal CM-fields of a given degree, one determines their possible Galois groups and tries to solve this problem for a given Galois group. However, there are too many groups of 2-power orders, e.g., 267 groups of order 64 and 2328 groups of order 128 (see [M], [HS], JNO, Wo, [TW, and [Ob]). It seems reasonable to try to get analytically sharper bounds for the degrees of normal CM-fields with class number one than the previously known ones, even with the assumption of the Generalized Riemann Hypothesis. Let $K$ be a CM-field of degree $2 n$ with maximal totally real subfield $k, D_{K}$ the absolute value of its discriminant, $\rho_{K}=D_{K}^{1 / 2 n}$ its root discriminant, and let $h_{K}^{-}=h_{K} / h_{k}$ be its relative class number, where $h_{K}$ and $h_{k}$ are the class number of $K$ and that of $k$, respectively. The purpose of this paper is to show the following.

Theorem 1. Let $K$ be a CM-field of degree $2 n$.

(1) We assume the Generalized Riemann Hypothesis and assume that $K$ is normal over $\mathbb{Q}$. If $n \geq 50$, then

$$
h_{K}^{-} \geq \frac{2}{6.69671} \cdot \frac{(1.06730)^{n}}{\log (2 n \cdot 3.77012)} \geq 1.30699>1 .
$$

Received by the editor January 19, 2005 and, in revised form, February 27, 2005.

2000 Mathematics Subject Classification. Primary 11R29, 11R42.

Key words and phrases. CM-fields, class numbers, relative class numbers, Dedekind zeta functions.

(C)2005 American Mathematical Society Reverts to public domain 28 years from publication 
If $h_{K}^{-}=1$, then $n \leq 48$ and $\rho_{K} \leq \alpha(n)$, where the $\alpha(n)$ 's are given in Table 6 below. If $50 \leq n \leq 83$, then $h_{K}^{-} \geq h(n)$, where the $h(n)$ 's are given in Table 5 below.

(2) We do not assume the Generalized Riemann Hypothesis, but assume that $K$ is normal over $\mathbb{Q}$. If $n \geq 109$, then

$$
h_{K}^{-} \geq \frac{(1.06136)^{n}}{n \cdot 5.98607} \geq 1.01035>1 .
$$

If $109 \leq n \leq 134$, then $h_{K}^{-} \geq h(n)$, where the $h(n)$ 's are given in Table 7 at the end of this paper.

(3) We assume the Generalized Riemann Hypothesis, but do not assume that $K$ is normal over $\mathbb{Q}$. If $n \geq 53$, then

$$
h_{K}^{-} \geq \frac{(1.08068)^{n}}{\sqrt{n}(6.17730)(1.43729)^{1 / \sqrt{n}}} \geq 1.29231>1 .
$$

If $53 \leq n \leq 88$, then $h_{K}^{-} \geq h(n)$, where the $h(n)$ 's are given in Table 8 at the end of this paper.

All imaginary abelian number fields with class number one are known by Yamamura (see [Y] and [CK1]). By many authors all normal CM-fields of degrees $<48$ with class number one are known. The class number one problem for normal CM-fields of degree 48 is partially solved. For full details see [LO1, [LOO, [LO2], [Lou1, Lef], LLO], [PYK], CK2], [P], LPCK], and [CK3]. Recently, it has been proved that there are no normal CM-fields of degree $\leq 96$ with class number one except for the possible fields of degree 64 or 96 (see [PK]). We remark that in [PK] the authors used the upper bound for root discriminant $\alpha(24)$ above to solve the class number one problem for the normal CM-fields of degree 48. Note that there are $223(=230-7)$ nonabelian groups of order 96 .

For a number field $M$ we let $O_{M}, \kappa_{M}$, and $\omega_{M}$ be its ring of algebraic integers, the residue of $\zeta_{M}(s)$ (the Dedekind zeta function of $M$ ) at $s=1$, and the number of roots of unity in $M$, respectively. For a CM-field $K$ we denote by $Q_{K} \in\{1,2\}$ its Hasse unit index. From the analytic class number formula we have

$$
h_{K}^{-}=\frac{Q_{K} \omega_{K}}{(2 \pi)^{n}} \sqrt{\frac{D_{K}}{D_{k}}} \frac{\kappa_{K}}{\kappa_{k}} \geq \frac{2 D_{K}^{1 / 4}}{(2 \pi)^{n}} \frac{\kappa_{K}}{\kappa_{k}},
$$

where $[K: \mathbb{Q}]=2 n([\mathrm{~W}]$.

To prove Theorem 1 we proceed as follows. Using Weil's explicit formula, we get explicit lower bounds for $D_{K}$ in Section 2 and upper bounds for $\kappa_{k}$ in Section 3. Using this Weil's formula Bessassi improved significantly the previously known bounds for $\kappa_{k}$. We take care of prime ideals of small norms when we deal with this Weil's formula, which allows us to improve upon Bessassi's upper bounds for $\kappa_{k}$. In Section 4 we give lower bounds for $\kappa_{K}$. When we do not assume the Generalized Riemann Hypothesis, we use Louboutin's bounds in [Lou3]. When we assume the Generalized Riemann Hypothesis, we use Bessassi's result in $[\mathbf{B}$, and what is more we take care of prime ideals of small norms. In Section 5 , using those bounds above we get explicit lower bounds for $h_{K}^{-}$. Ultimately Section 6 is devoted to the proof of Theorem 1 itself. 


\section{LOWER BOUNDS FOR $D_{K}$}

To begin with, we recall the following result deduced from Weil's explicit formula.

Proposition 2. Let $F$ be a real-valued even function with $F(0)=1$ for which the following conditions hold.

(i) The sum $\int_{0}^{\infty} F(x) \cosh (x / 2) d x$ exists.

(ii) The function $F$ is of bounded variation, the value in each point being the average of the limit to the right and the limit to the left.

(iii) The function $(1-F(x)) / x$ is also of bounded variation.

(iv) Assuming the Generalized Riemann Hypothesis, the Fourier transform of F is nonnegative. Without this hypothesis, the Fourier transform of $f(x)=$ $F(x) \cosh (x / 2)$ is nonnegative.

Let $k$ be a totally real number field of degree $n$ over $\mathbb{Q}$. Set

$$
I_{n}(F)=\frac{4}{n} \int_{0}^{\infty} F(x) \cosh (x / 2) d x+\int_{0}^{\infty} \frac{(1-F(x)) e^{x / 2}}{\sinh (x)} d x
$$

and $B_{n}(F)=\log \left(8 \pi e^{\gamma}\right)+(\pi / 2)-I_{n}(F)$. For a positive integer $i \geq 2$ we let $g_{i} \geq 0$ be the number of prime ideals $\mathfrak{p}$ in $k$ with $N_{k / \mathbb{Q}}(\mathfrak{p})=i$, and

$$
l_{i}(F)=\sum_{m \geq 1} \frac{\log i}{i^{m / 2}} F(m \log i) .
$$

Then we have

$$
\log D_{k} \geq n B_{n}(F)+2 \sum_{i \geq 2} g_{i} l_{i}(F) .
$$

Proof. See [Poi1, Propositions 4 and 5] and Section 3 in [B].

To get lower bounds for discriminants assuming the Generalized Riemann Hypothesis we choose

$$
0 \leq F(x)=F_{O, b_{O}}(x)= \begin{cases}\left(1-\left|\frac{x}{b_{O}}\right|\right) \cos \left(\pi x / b_{O}\right)+\frac{1}{\pi} \sin \left(\pi\left|x / b_{O}\right|\right) & \text { for }|x| \leq b_{O} \\ 0 & \text { for }|x|>b_{O}\end{cases}
$$

as chosen by Odlyzko ( $\mathrm{O} 2$ and $[$ Poi2]). Without assuming the Generalized Riemann Hypothesis it is known that Tartar's choice is the best one ([Poi1], Poi2], and $\mathrm{O} 2$ ):

$$
0 \leq F(x)=F_{T, b_{T}}(x)= \begin{cases}9\left(\frac{\sin \left(x / b_{T}\right)-\left(x / b_{T}\right) \cos \left(x / b_{T}\right)}{\left(x / b_{T}\right)^{3}}\right)^{2} / \cosh (x / 2) & \text { for } x \neq 0, \\ 1 & \text { for } x=0 .\end{cases}
$$

Here $b_{O}>0$ and $b_{T}>0$ will be chosen for each degree $n$ to give the best possible bound. For these $b_{O}$ and $b_{T}$ we let $B(n)=B_{n}\left(F_{O, b_{O}}\right)$ and $l_{i}=l_{i}\left(F_{O, b_{O}}\right)$ if we assume the Generalized Riemann Hypothesis, $B(n)=B_{n}\left(F_{T, b_{T}}\right)$ and $l_{i}=$ $l_{i}\left(F_{T, b_{T}}\right)$ otherwise. We can easily estimate $B(n)$, e.g., $B_{5}\left(F_{O, 3.13308}\right)=1.89381$, $B_{6}\left(F_{O, 3.51124}\right)=2.09779, B_{5}\left(F_{T, 1.00000}\right)=1.79442$, and $B_{6}\left(F_{T, 1.00000}\right)=2.04584$. We will need the fact that $B(5)>1$ and $B(6)>2$ in the proof of Lemma 7 below. Note that $n \mapsto B(n)$ is increasing, and that both $l_{i}\left(F_{O, b_{O}}\right)$ and $l_{i}\left(F_{T, b_{T}}\right)$ are always nonnegative. 


\section{UPPER BOUNDS FOR $\kappa_{k}$}

Usually an upper bound for $\kappa_{k}$ is obtained by first estimating $\zeta_{k}(\sigma)$ with $\sigma>$ 1. To estimate $\zeta_{k}(\sigma)$ from above we use Weil's formula with $F(x)=F_{b}(x)=$ $\left.\operatorname{sech}(x / 2) /\left(1+(x / b)^{2}\right)(\mathrm{B}]\right)$. Since the Fourier transform of $f_{b}(x)=F_{b}(x) \cosh (x / 2)$ $=1 /\left(1+(x / b)^{2}\right)$ is $\hat{f}_{b}(t)=\frac{1}{\sqrt{2 \pi}} \int_{-\infty}^{\infty} f_{b}(x) e^{-i t x} d x=\sqrt{\frac{\pi}{2}} b e^{-b|t|} \geq 0$, we can use Proposition 2 ,

For real $x>1$ and $t \geq 3$ a positive integer, we set

$$
\begin{gathered}
c_{0}(\sigma, b, x):=-\log \left(1-x^{-\sigma}\right) /\left(2 \sum_{m \geq 1} \frac{\log x}{x^{m / 2}} F_{b}(m \log x)\right), \\
c_{1}(\sigma, b, t):=\sup _{x \geq t} c_{0}(\sigma, b, x), \\
L_{i}(\sigma, b, t):=\left(c_{0}(\sigma, b, i)-c_{1}(\sigma, b, t)\right) l_{i}\left(F_{b}\right),
\end{gathered}
$$

and

$$
C_{2}(b, n):=B_{n}\left(F_{b}\right) .
$$

Remark 1. Since $t \geq 3$, our $c_{1}(\sigma, b, t)$ is smaller than Bessassi's $c_{1}(\sigma, b)=c_{1}(\sigma, b, 2)$ in [B, Theorem 4]. This allows us to improve upon Bessassi's upper bounds for $\kappa_{k}$ in Proposition 5 below. (See Remark 2 below.)

Lemma 3. Let $\sigma>1$ be given. We have

$$
\zeta_{k}(\sigma) \leq\left(\frac{D_{k}}{\exp \left(n C_{2}(b, n)\right)}\right)^{c_{1}(\sigma, b, t)} \prod_{i=2}^{t-1}\left[\exp \left(2 L_{i}(\sigma, b, t)\right)\right]^{g_{i}}
$$

for every positive integer $t \geq 3$.

Proof. We have

$$
\begin{array}{rlrl}
\log \zeta_{k}(\sigma) & =-\sum_{i \geq 2} g_{i} \log \left(1-i^{-\sigma}\right) & \\
& =2 \sum_{i \geq 2} g_{i} c_{0}(\sigma, b, i) l_{i}\left(F_{b}\right) & & \text { by }(3.1)) \\
& \leq 2 \sum_{i=2}^{t-1} g_{i} c_{0}(\sigma, b, i) l_{i}\left(F_{b}\right)+2 c_{1}(\sigma, b, t) \sum_{i \geq t} g_{i} l_{i}\left(F_{b}\right) & & \text { by }(3.2)) \\
& =2 \sum_{i=2}^{t-1} g_{i} L_{i}(\sigma, b, t)+2 c_{1}(\sigma, b, t) \sum_{i \geq 2} g_{i} l_{i}\left(F_{b}\right) & & (\text { by }(3.3)) \\
& \leq 2 \sum_{i=2}^{t-1} g_{i} L_{i}(\sigma, b, t)+c_{1}(\sigma, b, t)\left(\log D_{k}-n B_{n}\left(F_{b}\right)\right) & & (\text { by }(2.2)) \\
& =2 \sum_{i=2}^{t-1} g_{i} L_{i}(\sigma, b, t)+c_{1}(\sigma, b, t)\left(\log D_{k}-n C_{2}(b, n)\right) & & (\text { by }(3.4)) .
\end{array}
$$

The result follows.

Lemma 4. Let $k$ be a totally real number field of degree $n \geq 1$. If $\zeta_{k}(s)$ has no real zero in the range $1 / 2<\beta<1$, we set $E_{\sigma}=1$; if $\beta$ is any real zero of $\zeta_{k}(s)$ in this range, we set $E_{\sigma}=\frac{1-\beta}{\sigma-\beta}$. Set

$$
h(\sigma)=\pi^{-\sigma / 2} \Gamma(\sigma / 2) \quad(\sigma>1)
$$

and $\psi(\sigma)=\left(\Gamma^{\prime} / \Gamma\right)(\sigma)$. For $\tilde{\sigma}$ such that $\tilde{\sigma} \geq 1+\sigma / \sqrt{7+4 \sqrt{2}}$ and $\tilde{\sigma} \geq$ $\left(5+\sqrt{12 \sigma^{2}-5}\right) / 6$ if we do not assume the Generalized Riemann Hypothesis, and 
$\tilde{\sigma} \geq 1+(\sigma-1) / \sqrt{3}$ if we do assume the Generalized Riemann Hypothesis, set

$$
c_{3}(\tilde{\sigma}, n)=\frac{n}{4} \psi^{\prime}\left(\frac{\tilde{\sigma}}{2}\right)-\frac{1}{\tilde{\sigma}^{2}}-\frac{1}{(\tilde{\sigma}-1)^{2}} .
$$

Then, for $\sigma>1$ we have

$$
\kappa_{k}<E_{\sigma} \frac{\sigma(\sigma-1) \zeta_{k}(\sigma) D_{k}^{(\sigma-1) / 2} h^{n}(\sigma)}{\exp \left(\sigma(\sigma-1) c_{3}(\tilde{\sigma}, n) / 2\right)}
$$

Proof. See [01, Poi2, [W, Lemma 11.21], and [B, Lemmas 5 and 6].

Putting together Lemmas 3 and 4 we get an upper bound for $\kappa_{k}$ :

Proposition 5. Let $c_{1}=c_{1}(\sigma, b, t), C_{2}=C_{2}(b, n), h(\sigma), c_{3}=c_{3}(\tilde{\sigma}, n)$, and $E_{\sigma}$ be as above. Let

and let

$$
c_{4}=c_{4}(\sigma, b, t)=c_{1}(\sigma, b, t)+\frac{\sigma-1}{2}
$$

$$
C_{5}(n, b, t, \sigma, \tilde{\sigma})=\exp \left(\frac{1}{c_{4}}\left(c_{1} C_{2}+\frac{\sigma(\sigma-1)}{2} \frac{c_{3}}{n}-\frac{1}{n} \log (\sigma(\sigma-1))-\log h(\sigma)\right)\right) .
$$

(1) Let $k$ be a totally real number field of degree $n$. We have then

$$
\kappa_{k} \leq E_{\sigma}\left(\frac{D_{k}}{C_{5}(n, b, t, \sigma, \tilde{\sigma})^{n}}\right)^{c_{4}(\sigma, b, t)} \prod_{i=2}^{t-1}\left[\exp \left(2 L_{i}(\sigma, b, t)\right)\right]^{g_{i}}
$$

for every positive integer $t \geq 3$.

(2) Let $m \geq 1$ and $t \geq 3$ be given integers and let $b>0$ be given. Assume that for given $m, b$, and $t$ we have chosen $\sigma$ and $\tilde{\sigma}$. Set $c_{4}(m, b, t)=c_{4}(\sigma, b, t)$, $C_{5}(m, b, t)=C_{5}(m, b, t, \sigma, \tilde{\sigma})$, and $L_{i}(m, b, t)=L_{i}(\sigma, b, t)$. Then

$$
\kappa_{k} \leq E_{\sigma}\left(\frac{D_{k}}{C_{5}(m, b, t)^{n}}\right)^{c_{4}(m, b, t)} \prod_{i=2}^{t-1}\left[\exp \left(2 L_{i}(m, b, t)\right)\right]^{g_{i}}
$$

for any totally real number field $k$ of degree $n \geq m$.

Possible values for $c_{4}(\sigma, b, t)$ and $C_{5}(m, b, t, \sigma, \tilde{\sigma})$ for small degrees are given in Table 1 below. These values for $m \geq 108$ are unconditionally obtained and those for $48 \leq m \leq 83$ are obtained assuming the Generalized Riemann Hypothesis.

Proof. (1) To get possible values for $C_{5}(n, b, t, \sigma, \tilde{\sigma})$ and $c_{4}(\sigma, b, t)$ we proceed as follows. First, we fix $n$. Second, we fix $b$ and $t$. (In the proof of Theorem 1 below we will explain how to choose favorable values for $b$ and $t$ for a given $n$.) Third, we find $\sigma \geq 1.01$ having three properties at once: (i) $\sigma$ is as small as possible; (ii) $c_{4}(\sigma, b, t)$ is as small as possible; (iii) $2 \pi b c_{1}(\sigma, b, t)+\log (\sigma(\sigma-1)) \geq 0$. (For the reason why we want $\sigma \geq 1.01$, see the proof of Proposition 8 point (2) below.) Finally we choose $\tilde{\sigma}$ so that $c_{3}(\tilde{\sigma}, n)$ is as large as possible. For given $n, b, t$, and $\sigma$, the values $L_{i}(\sigma, b, t)$ can be easily computed.

(2) The function $n \mapsto C_{5}(n, b, t, \sigma, \tilde{\sigma})$ is increasing for given $b, t, \sigma$, and $\tilde{\sigma}$. Once we have chosen $b, t, \sigma$, and $\tilde{\sigma}$ for a given $m$, we have then $C_{5}(n, b, t, \sigma, \tilde{\sigma}) \geq$ $C_{5}(m, b, t)$. So,

$$
\kappa_{k} \leq E_{\sigma}\left(\frac{D_{k}}{C_{5}(m, b, t)^{n}}\right)^{c_{4}(m, b, t)} \prod_{i=2}^{t-1}\left[\exp \left(2 L_{i}(m, b, t)\right)\right]^{g_{i}}
$$

for any totally real number field $k$ of degree $n \geq m$. 
TABLE 1.

\begin{tabular}{|c|c|c|c|c|c|c|}
\hline$m$ & $b$ & $t$ & $\sigma$ & $\tilde{\sigma}$ & $c_{4}(\sigma, b, t)$ & $C_{5}(m, b, t, \sigma, \tilde{\sigma})$ \\
\hline 134 & 8.866 & 47 & 1.0162 & 1.2865 & 0.08181 & 54.799 \\
130 & 8.678 & 45 & 1.0167 & 1.2868 & 0.08310 & 54.604 \\
120 & 8.480 & 43 & 1.0172 & 1.2872 & 0.08452 & 54.361 \\
110 & 8.163 & 40 & 1.0181 & 1.2879 & 0.08689 & 53.967 \\
109 & 8.163 & 40 & 1.0181 & 1.2879 & 0.08689 & 53.961 \\
108 & 8.163 & 40 & 1.0181 & 1.2879 & 0.08689 & 53.954 \\
\hline 83 & 12.103 & 101 & 1.0107 & 1.2882 & 0.06485 & 56.587 \\
80 & 12.103 & 101 & 1.0107 & 1.2928 & 0.06485 & 56.561 \\
72 & 12.103 & 101 & 1.0107 & 1.3064 & 0.06485 & 56.483 \\
70 & 12.103 & 101 & 1.0107 & 1.3102 & 0.06485 & 56.462 \\
64 & 11.890 & 96 & 1.0110 & 1.3226 & 0.06573 & 56.297 \\
60 & 10.740 & 73 & 1.0126 & 1.3319 & 0.07096 & 55.627 \\
50 & 8.678 & 45 & 1.0167 & 1.3601 & 0.08310 & 53.851 \\
49 & 8.678 & 45 & 1.0167 & 1.3634 & 0.08310 & 53.832 \\
48 & 8.480 & 43 & 1.0172 & 1.3668 & 0.08452 & 53.608 \\
\hline
\end{tabular}

Remark 2. When $n=83$, Bessassi obtained $C_{5}(83, b, 2, \sigma, \tilde{\sigma})=38.9255$ and $c_{4}(\sigma, b, 2)$ $=0.36705$ with $b=4.78685, \sigma=1.01114$, and $\tilde{\sigma}=1.28823$ in [B. Table 4]. However we obtained $C_{5}(83, b, t, \sigma, \tilde{\sigma})=56.58718 \cdots$ and $c_{4}(\sigma, b, t)=0.0648497 \cdots$ with $b=12.103, \sigma=1.0107405 \cdots, \tilde{\sigma}=1.288211 \cdots$, and $t=101$. This improvement on upper bounds for $\kappa_{k}$ and Lemma 7 yield that $h_{K}^{-} \geq 48986$ if $[K: \mathbb{Q}]=2 \cdot 83$ in the proof of Theorem 1 point (1) below.

\section{LOWER BOUNDS FOR $\kappa_{K}$}

Proposition 6. (1) Let $m \geq 1$ and $\rho>e$ be given. Assume the Generalized Riemann Hypothesis. For any normal number field $K$ of degree $n \geq m$ and of root discriminant $\rho_{K} \geq \rho$ we have

$$
\kappa_{K} \geq \frac{1}{c_{6}(m, \rho) \log \log D_{K}} \prod_{i=2}^{t-1}\left(\frac{1}{1-i^{-2}}\right)^{g_{i}},
$$

where $t \geq 3$ is a positive integer and the $c_{6}(m, \rho)$ 's can be taken as in Table 2 .

(2) For any totally imaginary number field $K$ of degree $\geq 10$ and root discriminant $\rho_{K} \geq 2 \pi^{2}$, we have unconditionally

$$
\kappa_{K} \geq \begin{cases}\frac{1}{c e^{1 /(2 c)} \log D_{K}} & \text { if } \zeta_{K}\left(1-1 /\left(c \log D_{K}\right)\right) \leq 0, \\ \frac{1-\beta}{2 e^{1 /(2 c)}} & \text { if } \zeta_{K}(\beta) \leq 0 \text { with } 1-1 /\left(c \log D_{K}\right) \leq \beta<1,\end{cases}
$$

where $c=(2+\sqrt{3}) / 4$.

TABLE 2 .

\begin{tabular}{|c|c|c|c|c|c|c|c|}
\hline$m$ & 98 & 96 & 80 & 64 & 60 & 48 & 46 \\
\hline$\rho$ & 50.29 & 50.71 & 54.98 & 61.90 & 64.37 & 75.08 & 77.61 \\
\hline$c_{6}(m, \rho)$ & 6.6705 & 6.6778 & 6.7432 & 6.8245 & 6.8482 & 6.9303 & 6.9460 \\
\hline \hline$m$ & 40 & 36 & 32 & 30 & 20 & 16 & 10 \\
\hline$\rho$ & 87.36 & 96.55 & 109.3 & 117.7 & 211.0 & 324.7 & 1163 \\
\hline$c_{6}(m, \rho)$ & 6.9972 & 7.0355 & 7.0779 & 7.1008 & 7.2384 & 7.3076 & 7.4320 \\
\hline
\end{tabular}


(3) [B, Theorem 14] For any number field $K$ different from $\mathbb{Q}$ for which the Generalized Riemann Hypothesis for $\zeta_{K}(s)$ holds true, we have

$$
\kappa_{K} \geq \frac{e^{-3 / 2}}{\sqrt{\log D_{K}}} \exp \left(\frac{-1}{\sqrt{\log D_{K}}}\right) .
$$

Proof. (1) We have

$$
\kappa_{K}=\lim _{s \rightarrow 1}(s-1) \zeta_{K}(s)=\lim _{s \rightarrow 1} \frac{\zeta_{K}(s)}{\zeta(s)}=\prod_{p} E(p)=M(Q) R(Q) T(Q),
$$

where

$$
\begin{aligned}
& E(p)=\left(1-p^{-1}\right) \prod_{\mathcal{P}_{K} \mid p}\left(1-N_{K / \mathbb{Q}}\left(\mathcal{P}_{K}\right)^{-1}\right)^{-1} \geq\left(1-p^{-1}\right) \prod_{\mathcal{P}_{k} \mid p}\left(1-N_{k / \mathbb{Q}}\left(\mathcal{P}_{k}\right)^{-2}\right)^{-1}, \\
& M(Q)=\prod_{p \leq Q} E(p), R(Q)=\prod_{\substack{p>Q \\
p>\operatorname{comified}}} E(p), \text { and } T(Q)=\prod_{\substack{p>Q \\
p \text { nonramified }}} E(p) \text {. For an }
\end{aligned}
$$

estimate of $T(Q)$ and a lower bound for $R(Q)$ we use B, Lemma 11 and (26)]. For $M(Q)$, we choose $t$ and $Q$ with $t \leq Q$ so that

$$
\begin{aligned}
M(Q) & =\prod_{p \leq Q} E(p) \\
& \geq \prod_{p \leq Q}\left(1-p^{-1}\right) \prod_{i=2}^{t-1}\left(1-i^{-2}\right)^{-g_{i}} \\
& \geq \frac{1}{e^{\gamma} \log Q}\left(1-\frac{1}{2(\log Q)^{2}}\right) \prod_{i=2}^{t-1}\left(1-i^{-2}\right)^{-g_{i}} .
\end{aligned}
$$

The remainder of the proof is the same as that of [B, Corollary 13].

(2) See [Lou2, Theorem 1] and [B, Theorem 16].

\section{LOWER BOUNDS FOR $h_{K}^{-}$}

To begin with we prove the following,

Lemma 7. Let $K$ be a $C M$-field of degree $2 n \geq 2 m$ with maximal real subfield $k$, $t \geq 3$ a given positive integer, and let $\varepsilon \geq 0.05$ be a positive real number. Then we have

(1) $D_{K} \geq \exp (2 n B(m)) \prod_{i=2}^{t-1}\left(\exp \left(4 l_{i}\right)\right)^{g_{i}}$,

(2) $\frac{D_{K}{ }^{\varepsilon}}{\log \log D_{K}} \geq \frac{\exp (2 \varepsilon n B(m))}{\log (2 n B(m))} \prod_{i=2}^{t-1}\left(\exp \left(4 \varepsilon l_{i}\right) /\left(1+\frac{2 l_{i}}{m B(m)}\right)\right)^{g_{i}}$ if $m \geq 5$,

(3) $\frac{D_{K}{ }^{\varepsilon}}{\log D_{K}} \geq \frac{\exp (2 \varepsilon n B(m))}{2 n B(m)} \prod_{i=2}^{t-1}\left(\exp \left(4 \varepsilon l_{i}\right) /\left(1+\frac{2 l_{i}}{m B(m)}\right)\right)^{g_{i}}$ if $m \geq 6$,

(4) $\frac{D_{K} \varepsilon}{\left(\log D_{K}\right)^{1 / 2}} \geq \frac{\exp (2 \varepsilon n B(m))}{(2 n B(m))^{1 / 2}} \prod_{i=2}^{t-1}\left(\exp \left(4 \varepsilon l_{i}\right) /\left(1+\frac{l_{i}}{m B(m)}\right)\right)^{g_{i}}$ if $m \geq 5$.

Proof. For (1), by Proposition 2 and since $n \mapsto B(n)$ increases with $n$, we have

$$
\log D_{K} \geq 2 \log D_{k} \geq 2 n B(n)+4 \sum_{i \geq 2} g_{i} l_{i} \geq 2 n B(m)+4 \sum_{i=2}^{t-1} g_{i} l_{i} .
$$

(2) Now, using $A^{1+\delta} \geq A e^{\delta} \geq A(1+\delta)$ for $A \geq e$ and $\delta \geq 0$, we have

$$
\log \left(A+4 \sum_{i=2}^{t-1} g_{i} l_{i}\right) \leq\left(1+\sum_{i=2}^{t-1} \frac{4 g_{i} l_{i}}{A}\right) \log A \leq\left(\prod_{i=2}^{t-1}\left(1+\frac{4 l_{i}}{A}\right)^{g_{i}}\right) \log A
$$


for $A=2 n B(m) \geq e$. Since $2 n \geq 2 m \geq 10$ implies $A=2 n B(m) \geq 10 B(m) \geq$ $10 B(5) \geq 10 \geq e$ (see the end of Section 2) and since $x \mapsto x^{\varepsilon} / \log \log x$ is increasing for $x \geq e^{10}$ and $\varepsilon \geq 0.05$, using (5.1) we obtain

$$
\frac{D_{K}^{\varepsilon}}{\log \log D_{K}} \geq \frac{\exp \left(\varepsilon\left(A+4 \sum_{i=2}^{t-1} g_{i} l_{i}\right)\right)}{\log \left(A+4 \sum_{i=2}^{t-1} g_{i} l_{i}\right)},
$$

and (2) follows, by (5.2). The proofs of (3) and (4) are similar. For (3) we use the fact that $x \mapsto x^{\varepsilon} / \log x$ is increasing if $x \geq e^{20}$ and $\varepsilon \geq 0.05$.

Combining Propositions $\left[5\right.$ and $\left[6\right.$ and Lemma 7 we get lower bounds for $h_{K}^{-}$as follows.

Proposition 8. Let $m \geq 1$ and $t \geq 3$ be given integers and let $b>0$ be given. Set

$$
C_{7}(n, b, t)=(2 \pi)^{-1}\left(C_{5}(n, b, t) \exp (-B(n))\right)^{c_{4}(n, b, t)} \exp (B(n) / 2) .
$$

Suppose that $c_{4}(n, b, t) \leq 0.4$ for every $n \geq 5$.

(1) Assume the Generalized Riemann Hypothesis. Let $\rho>e$ be given. For any normal $C M$-field $K$ of degree $2 n \geq 2 m \geq 10$ and root discriminant $\rho_{K} \geq \rho$ we have

$$
h_{K}^{-} \geq \frac{2}{c_{6}(2 m, \rho)} \frac{C_{7}(m, b, t)^{n}}{\log (2 n B(m))} \prod_{i=2}^{t-1}\left(\frac{\exp \left(\left(1-2 c_{4}(m, b, t)\right) l_{i}\right)}{\left(1+\frac{2 l_{i}}{m B(m)}\right)\left(1-i^{-2}\right) \exp \left(2 L_{i}(m, b, t)\right)}\right)^{g_{i}} .
$$

(2) Set $c=(2+\sqrt{3}) / 4=0.93301 \cdots$. For any normal $C M$-field $K$ of degree $2 n \geq 2 m \geq 100$ we have unconditionally

$$
h_{K}^{-} \geq \frac{C_{7}(m, b, t)^{n}}{c e^{1 /(2 c)} n B(m)} \prod_{i=2}^{t-1}\left(\frac{\exp \left(\left(1-2 c_{4}(m, b, t)-\frac{2}{m}\right) l_{i}\right)}{\exp \left(2 L_{i}(m, b, t)\right)}\right)^{g_{i}} .
$$

(3) Assume the Generalized Riemann Hypothesis. For any CM-field K (not necessarily normal over $\mathbb{Q}$ ) of degree $2 n \geq 2 m \geq 10$ we have

$$
h_{K}^{-} \geq \frac{2 C_{7}(m, b, t)^{n}}{e^{3 / 2}(2 n B(m))^{1 / 2}(\exp (1 / \sqrt{2 B(m)}))^{1 / \sqrt{n}}} \prod_{i=2}^{t-1}\left(\frac{\exp \left(\left(1-2 c_{4}(m, b, t)\right) l_{i}\right)}{\left(1+\frac{l_{i}}{m B(m)}\right) \exp \left(2 L_{i}(m, b, t)\right)}\right)^{g_{i}} .
$$

Proof. According to (1.4) we have $h_{K}^{-} \geq 2\left(D_{K}^{1 / 4} /(2 \pi)^{n}\right)\left(\kappa_{K} / \kappa_{k}\right)$.

(1) By Propositions 5 and 6 point (1), and since $D_{K} \geq D_{k}{ }^{2}$,

$h_{K}^{-} \geq \frac{2}{c_{6}(2 m, \rho)}\left(\frac{C_{5}(m, b, t)^{c_{4}(m, b, t)}}{2 \pi}\right)^{n} \frac{D_{K}^{\frac{1}{4}-\frac{1}{2} c_{4}(m, b, t)}}{\log \log D_{K}} \prod_{i=2}^{t-1}\left(\frac{1}{\left(1-i^{-2}\right) \exp \left(2 L_{i}(m, b, t)\right)}\right)^{g_{i}}$.

By applying Lemma 7 point (2) with $\varepsilon=\frac{1}{4}-\frac{1}{2} c_{4}(m, b, t)$, we get (5.3).

(2) Set $I=\left[1-1 /\left(c \log D_{K}\right), 1\right)$. We consider the following three cases.

(i) If $\zeta_{k}(s)$ has a zero $\beta \in I$, then $\zeta_{K}(\beta)=0$. By Propositions 5 and 6 point (2), and since $(1-\beta) / E_{\sigma}=\sigma-\beta>\sigma-1$,

$$
h_{K}^{-} \geq \frac{(\sigma-1)}{e^{1 /(2 c)}}\left(\frac{C_{5}(m, b, t)^{c_{4}(m, b, t)}}{2 \pi}\right)^{n} D_{K}^{\frac{1}{4}-\frac{1}{2} c_{4}(m, b, t)} \prod_{i=2}^{t-1}\left(\frac{1}{\exp \left(2 L_{i}(m, b, t)\right)}\right)^{g_{i}} .
$$


By applying Lemma 7 point (1), we have

$$
h_{K}^{-} \geq \frac{(\sigma-1)}{e^{1 /(2 c)}} C_{7}(m, b, t)^{n} \prod_{i=2}^{t-1}\left(\frac{\exp \left(\left(1-2 c_{4}(m, b, t)\right) l_{i}\right)}{\exp \left(2 L_{i}(m, b, t)\right)}\right)^{g_{i}} .
$$

Here, $\sigma$ was given in Proposition 5 .

(ii) If $\zeta_{k}(s)$ has no zero $\beta$ in $I$ and $\zeta_{K}(s)$ has a simple zero in $I$, then by $\underline{S}$, Theorem 3] and [B, Theorem 17] there exists an imaginary quadratic subfield $F$ of $K$ such that $\zeta_{F}(\beta)=0$ and

We have

$$
1-\beta>\frac{6}{\pi} \frac{1}{\sqrt{D_{F}}}>\frac{6}{\pi} \frac{1}{D_{K}^{1 /(2 n)}} .
$$

$$
\begin{aligned}
h_{K}^{-} & \geq 2 \frac{D_{K}^{1 / 4}}{(2 \pi)^{n}} \frac{1}{E_{\sigma}}\left(\frac{C_{5}(m, b, t)^{n}}{D_{k}}\right)^{c_{4}(m, b, t)} \frac{1-\beta}{2 e^{1 /(2 c)}} \prod_{i=2}^{t-1}\left(\frac{1}{\exp \left(2 L_{i}(m, b, t)\right)}\right)^{g_{i}} \\
& \geq \frac{6}{\pi e^{1 /(2 c)}}\left(\frac{C_{5}(m, b, t)^{c_{4}(m, b, t)}}{2 \pi}\right)^{n} D_{K}^{\frac{1}{4}-\frac{1}{2} c_{4}(m, b, t)-\frac{1}{2 n}} \prod_{i=2}^{t-1}\left(\frac{1}{\exp \left(2 L_{i}(m, b, t)\right)}\right)^{g_{i}} .
\end{aligned}
$$

By applying Lemma 7 point (1), we have

$$
h_{K}^{-} \geq \frac{6}{\pi} \frac{C_{7}(m, b, t)^{n}}{e^{1 /(2 c)} e^{B(m)}} \prod_{i=2}^{t-1}\left(\frac{\exp \left(\left(1-2 c_{4}(m, b, t)-2 / m\right) l_{i}\right)}{\exp \left(2 L_{i}(m, b, t)\right)}\right)^{g_{i}} .
$$

(iii) If $\zeta_{k}(s)$ has no zero in $I$ and $\zeta_{K}(s)$ has no simple zero in $I$, then either $\zeta_{K}(s)$ has no zero at all in $I$ or $\zeta_{K}(s)$ has a double zero in $I$. This is because $\zeta_{K}(s)$ has at most two zeros with multiplicity in $I$ by [LLO, Lemma 15]. Then $\zeta_{K}\left(1-1 /\left(c \log D_{K}\right)\right) \leq 0$ and

$$
h_{K}^{-} \geq \frac{2}{c e^{1 /(2 c)}}\left(\frac{C_{5}(m, b, t)^{c_{4}(m, b, t)}}{2 \pi}\right)^{n} \frac{D_{K}^{\frac{1}{4}-\frac{1}{2} c_{4}(m, b, t)}}{\log D_{K}} \prod_{i=2}^{t-1}\left(\frac{1}{\exp \left(2 L_{i}(m, b, t)\right)}\right)^{g_{i}}
$$

by Propositions 5 and 6 point (2).

By applying Lemma 7 point (3) with $\varepsilon=\frac{1}{4}-\frac{1}{2} c_{4}(m, b, t)$, we have

$$
h_{K}^{-} \geq \frac{C_{7}(m, b, t)^{n}}{c e^{1 /(2 c)} n B(m)} \prod_{i=2}^{t-1}\left(\frac{\exp \left(\left(1-2 c_{4}(m, b, t)\right) l_{i}\right)}{\left(1+\frac{2 l_{i}}{m B(m)}\right) \exp \left(2 L_{i}(m, b, t)\right)}\right)^{g_{i}} .
$$

Now, we compare the following three terms:

$$
(\sigma-1), \quad \frac{6}{\pi e^{B(m)}} \prod_{i=2}^{t-1}\left(\frac{1}{\exp \left(2 l_{i} / m\right)}\right)^{g_{i}}, \quad \frac{1}{c n B(m)} \prod_{i=2}^{t-1}\left(\frac{1}{1+\frac{2 l_{i}}{m B(m)}}\right)^{g_{i}} .
$$

Notice that $\sigma-1>1 / 100$ (see Proposition 5 and Table 1), that $5.372 \cdots=$ $\log \left(8 \pi e^{\gamma}\right)+\frac{\pi}{2} \geq B(m) \geq B_{50}\left(F_{T, b_{T}}\right)=3.524 \cdots>3$ with $b_{T}=2.44976$, and that

$$
\frac{6}{\pi e^{B(m)}}>\frac{6}{\pi e^{5.38}}>\frac{1}{120}>\frac{1}{135}=\frac{1}{0.9 \cdot 50 \cdot 3}>\frac{1}{\mathrm{cnB} B(m)}
$$

for $m \geq 50$. Since $\exp \left(2 l_{i} / m\right) \geq 1+2 l_{i} / m \geq 1+2 l_{i} /(m B(m))$, it follows that all three terms above are greater than or equal to

$$
\frac{1}{c n B(m)} \prod_{i=2}^{t-1}\left(\frac{1}{\exp \left(2 l_{i} / m\right)}\right)^{g_{i}} \text {. }
$$

The result follows. 
(3) By Propositions 5 and 6 point (3),

$$
\begin{aligned}
h_{K}^{-} \geq \frac{2}{e^{3 / 2}}\left(\frac{C_{5}(m, b, t)^{c_{4}(m, b, t)}}{2 \pi}\right)^{n} \frac{D_{K}^{\frac{1}{4}-\frac{1}{2} c_{4}(m, b, t)}}{\left(\log D_{K}\right)^{1 / 2}} \\
\quad \times \exp \left(\frac{-1}{\left(\log D_{K}\right)^{1 / 2}}\right) \prod_{i=2}^{t-1}\left(\frac{1}{\exp \left(2 L_{i}(m, b, t)\right)}\right)^{g_{i}} .
\end{aligned}
$$

By Lemma 7 point (4) with $\varepsilon=\frac{1}{4}-\frac{1}{2} c_{4}(m, b, t)$, we have

$$
\frac{D_{K}^{\frac{1}{4}-\frac{1}{2} c_{4}(m, b, t)}}{\left(\log D_{K}\right)^{1 / 2}} \geq \frac{\exp \left(2 n B(m)\left(\frac{1}{4}-\frac{1}{2} c_{4}(m, b, t)\right)\right)}{(2 n B(m))^{1 / 2}} \prod_{i=2}^{t-1}\left(\frac{\exp \left(\left(1-2 c_{4}(m, b, t)\right) l_{i}\right)}{1+\frac{l_{i}}{m B(m)}}\right)^{g_{i}},
$$

and by Lemma 7 point (1), we have

$$
\exp \left(\frac{-1}{\left(\log D_{K}\right)^{1 / 2}}\right) \geq \exp \left(\frac{-1}{(2 n B(m))^{1 / 2}}\right) .
$$

The result follows.

\section{Proof of Theorem 1}

6.1. Proof of Theorem 1 point (1). Let $K$ be a normal CM-field of degree $2 n \geq 2 m \geq 10$. For $i<t$, we set

$$
M(m, i, b, t)=\frac{\exp \left(\left(1-2 c_{4}(m, b, t)\right) l_{i}\right)}{\left(1+\frac{2 l_{i}}{m B(m)}\right)\left(1-i^{-2}\right) \exp \left(2 L_{i}(m, b, t)\right)}
$$

if $i$ is a power of a prime, $M(m, i, b, t)=1$ otherwise. If for a given $m$ we have $M(m, i, b, t) \geq 1$ for $2 \leq i<t$, then, according to (5.3), for any normal CM-field $K$ of degree $2 n \geq 2 m \geq 10$ with $\rho_{K} \geq \rho$ we have

$$
h_{K}^{-} \geq \frac{2}{c_{6}(2 m, \rho)} \frac{C_{7}(m, b, t)^{n}}{\log (2 n B(m))} .
$$

To find favorable values for $b$ and $t$ that maximize this right-hand side we proceed as follows. First, we fix $m$, say $m=83$. Using Proposition 2 with $F(x)=F_{O, b_{O}}(x)$ we get $\rho_{K} \geq \rho:=54.88741$. We get $B(83)=4.00528 \cdots$ with $F(x)=F_{O, b_{O}}(x)$ and $c_{6}(166, \rho)=6.43518 \cdots$. Second, we fix $b$, say $b=2$. Third, we let $t=3$ and choose $\sigma$ and $\tilde{\sigma}$ as in the proof of Proposition [5. We compute $c_{4}(83,2,3)=$ $0.37137 \cdots<0.4, C_{5}(83,2,3)=28.47193 \cdots$, and $C_{7}(83,2,3)=0.92404 \cdots$ and verify that $M(83,2,2,3)=1.53445 \cdots \geq 1$. This yields

$$
h_{K}^{-} \geq \frac{2}{6.43519} \frac{(0.92404)^{n}}{\log (8.01057 n)} \geq 0.00006 .
$$

Now we repeat the third step with $b=2$ for all $t$ with $4 \leq t \leq 101$ and observe the following.

(a) For all $t$ with $3 \leq t \leq 101$ we always have $c_{4}(83,2, t) \leq 0.4$.

(b) For any given $t$ with $3 \leq t \leq 101$ we have $M(83, i, 2, t) \geq 1$ for every $i$ with $2 \leq i<t$.

(c) $C_{7}(83,2, t)$ is increasing for $3 \leq t \leq 54$ and $1.0196596 \cdots=C_{7}(83,2,54)>$ $C_{7}(83,2,55)=1.0196589 \cdots$. 
Hence we get

$$
h_{K}^{-} \geq \frac{2}{6.43519} \frac{(1.01965)^{n}}{\log (8.01057 n)} \geq 0.24045 .
$$

We add 1 to $b$ and repeat the third step with $b=3$ for all $t$ with $3 \leq t \leq 101$. We verify that (a) and (b) are satisfied, and $C_{7}(83,3, t)$ is increasing for $3 \leq t \leq 87$ and $1.1152586 \cdots=C_{7}(83,3,87)>C_{7}(83,3,88)=1.1152514 \cdots$. At this time we get

$$
h_{K}^{-} \geq \frac{2}{6.43519} \frac{(1.11525)^{n}}{\log (8.01057 n)} \geq 408.768 .
$$

We repeat the second and the third step by adding 1 to $b$ until $b=20$. For a given $b$, if $C_{7}(83, b, t)$ is increasing for all $t$ with $3 \leq t \leq 101$, then we take $C_{7}(83, b, 101)$. It happens when $b \in\{13,14,15,16,17,18,19,20\}$. We verify that the value $C_{7}(83,12,101)=1.18138 \cdots$ is the largest among all computed $C_{7}(83, b, t)$ 's. We continue this process for all $b=12+\frac{1}{10} k$ with integers $k$ with $-10<k<10$. We verify that the value $C_{7}(83,12.1,101)=1.18144 \cdots$ is the largest among all computed $C_{7}(83, b, t)$ 's. For $b=12.1+\frac{1}{100} k$ with $-10<k<10$, we repeat the computations and obtain the largest value $C_{7}(83,12.10,101)=1.18144 \cdots$. Similarly we get the value $C_{7}(83,12.103,101)=1.18145 \cdots$, which is the largest for all $b=12.10+\frac{1}{1000} k$ with $-10<k<10$. We did not compute $C_{7}(83, b, t)$ with $b=12.103+10^{-4} k$ with $-10<k<10$. This is because there are negligible changes in $C_{7}(83, b, t)$ 's, $3 \leq t \leq 101$ for a given $b$ with the precision of $10^{-3}$. We conclude that for any normal CM-field $K$ of degree $2 n \geq 2 \cdot 83$,

$$
h_{K}^{-} \geq \frac{2}{6.43519} \frac{(1.18145)^{n}}{\log (8.01057 n)} \geq 48984 .
$$

We do the same computations for every $m$ with $49 \leq m \leq 83$. Our computational results are summarized in Tables [3, 4, and 5. The values $b, t$, and $C_{7}(n, b, t)$ given in Table 5 are the most favorable among our computational results.

TABLE 3 . Lower bounds for $h_{K}^{-}$when $n=83$ and $b=12.103$

\begin{tabular}{|c|c|c||c|c|c|}
\hline$t$ & $C_{7}(83,12.103, t)$ & $h_{K}^{-} \geq$ & $t$ & $C_{7}(83,12.103, t)$ & $h_{K}^{-} \geq$ \\
\hline 2 & 0.95408 & 0.00096 & 40 & 1.17115 & 28682 \\
\hline 3 & 1.03532 & 0.85265 & 60 & 1.17639 & 34303 \\
\hline 4 & 1.07183 & 15.136 & 80 & 1.17938 & 42349 \\
\hline 10 & 1.13531 & 1795.0 & 100 & 1.18136 & 48675 \\
\hline 20 & 1.15796 & 9250.1 & 101 & 1.18145 & 48984 \\
\hline
\end{tabular}

TABLE 4. Lower bounds for $h_{K}^{-}$when $n=50$ and $b=8.678$

\begin{tabular}{|c|c|c||c|c|c|}
\hline$t$ & $C_{7}(50,8.678, t)$ & $h_{K}^{-} \geq$ & $t$ & $C_{7}(50,8.678, t)$ & $h_{K}^{-} \geq$ \\
\hline 2 & 0.88222 & 0.00009 & 43 & 1.06685 & 1.27972 \\
\hline 3 & 0.95360 & 0.00468 & 44 & 1.06708 & 1.29359 \\
\hline 4 & 0.98514 & 0.02381 & 45 & 1.06730 & 1.30699 \\
\hline 10 & 1.03835 & 0.33047 & 46 & 1.06715 & 1.29784 \\
\hline 20 & 1.05627 & 0.77749 & 47 & 1.06701 & 1.28935 \\
\hline 30 & 1.06268 & 1.05213 & 48 & 1.06687 & 1.28092 \\
\hline 40 & 1.06611 & 1.23608 & 49 & 1.06673 & 1.27254 \\
\hline
\end{tabular}


TABLE 5. Lower bounds for $h_{K}^{-}$

\begin{tabular}{|c|c|c|c|c|c|c|c|}
\hline$n$ & $\rho: \rho_{K} \geq \rho$ & $B(n)$ & $c_{6}(2 n, \rho)$ & $b$ & $t$ & $C_{7}(n, b, t)$ & $h(n): h_{K}^{-} \geq h(n)$ \\
\hline 83 & 54.887 & 4.0053 & 6.4352 & 12.103 & 101 & 1.1814 & 48984 \\
\hline 82 & 54.608 & 4.0002 & 6.4408 & 12.103 & 101 & 1.1788 & 34577 \\
\hline 81 & 54.327 & 3.9950 & 6.4465 & 12.103 & 101 & 1.1761 & 24457 \\
\hline 80 & 54.041 & 3.9898 & 6.4523 & 12.103 & 101 & 1.1734 & 17311 \\
\hline 79 & 53.752 & 3.9844 & 6.4582 & 12.103 & 101 & 1.1707 & 12279 \\
\hline 78 & 53.460 & 3.9789 & 6.4642 & 12.103 & 101 & 1.1679 & 8719.0 \\
\hline 77 & 53.164 & 3.9734 & 6.4703 & 12.103 & 101 & 1.1650 & 6201.3 \\
\hline 76 & 52.864 & 3.9677 & 6.4765 & 12.103 & 101 & 1.1622 & 4415.7 \\
\hline 75 & 52.561 & 3.9620 & 6.4829 & 12.103 & 101 & 1.1592 & 3150.2 \\
\hline 74 & 52.253 & 3.9561 & 6.4893 & 12.103 & 101 & 1.1563 & 2250.6 \\
\hline 73 & 51.942 & 3.9501 & 6.4959 & 12.103 & 101 & 1.1533 & 1611.3 \\
\hline 72 & 51.626 & 3.9440 & 6.5026 & 12.103 & 101 & 1.1502 & 1154.7 \\
\hline 71 & 51.306 & 3.9378 & 6.5095 & 12.103 & 101 & 1.1471 & 828.99 \\
\hline 70 & 50.982 & 3.9315 & 6.5165 & 12.103 & 101 & 1.1439 & 596.26 \\
\hline 69 & 50.654 & 3.9250 & 6.5237 & 12.103 & 101 & 1.1407 & 429.46 \\
\hline 68 & 50.321 & 3.9184 & 6.5310 & 12.019 & 99 & 1.1374 & 309.80 \\
\hline 67 & 49.983 & 3.9117 & 6.5384 & 12.019 & 99 & 1.1340 & 223.99 \\
\hline 66 & 49.640 & 3.9048 & 6.5460 & 12.019 & 99 & 1.1306 & 162.25 \\
\hline 65 & 49.293 & 3.8978 & 6.5538 & 11.890 & 96 & 1.1272 & 117.69 \\
\hline 64 & 48.940 & 3.8906 & 6.5618 & 11.890 & 96 & 1.1237 & 85.605 \\
\hline 63 & 48.582 & 3.8833 & 6.5699 & 11.757 & 93 & 1.1201 & 62.408 \\
\hline 62 & 48.219 & 3.8758 & 6.5783 & 10.853 & 75 & 1.1164 & 45.633 \\
\hline 61 & 47.851 & 3.8681 & 6.5868 & 10.740 & 73 & 1.1127 & 33.435 \\
\hline 60 & 47.477 & 3.8602 & 6.5956 & 10.740 & 73 & 1.1090 & 24.566 \\
\hline 59 & 47.097 & 3.8522 & 6.6045 & 10.440 & 68 & 1.1052 & 18.092 \\
\hline 58 & 46.711 & 3.8440 & 6.6137 & 10.440 & 68 & 1.1013 & 13.359 \\
\hline 57 & 46.318 & 3.8356 & 6.6231 & 9.839 & 59 & 1.0973 & 9.8912 \\
\hline 56 & 45.920 & 3.8269 & 6.6328 & 9.839 & 59 & 1.0932 & 7.3451 \\
\hline 55 & 45.515 & 3.8181 & 6.6427 & 9.693 & 57 & 1.0891 & 5.4688 \\
\hline 54 & 45.103 & 3.8090 & 6.6530 & 9.383 & 53 & 1.0849 & 4.0813 \\
\hline 53 & 44.685 & 3.7996 & 6.6634 & 9.218 & 51 & 1.0806 & 3.0566 \\
\hline 52 & 44.259 & 3.7901 & 6.6742 & 9.218 & 51 & 1.0763 & 2.2956 \\
\hline 51 & 43.825 & 3.7802 & 6.6853 & 9.218 & 51 & 1.0718 & 1.7285 \\
\hline 50 & 43.385 & 3.7701 & 6.6968 & 8.678 & 45 & 1.0673 & 1.3069 \\
\hline 49 & 42.936 & 3.7597 & 6.7085 & 8.678 & 45 & 1.0626 & 0.9912 \\
\hline & & & & & & & \\
\hline
\end{tabular}

Since the complex conjugation is in the center of the Galois group $G(K / \mathbb{Q})$, every normal CM-field of degree 98 is an imaginary abelian number field. Hence its relative class number is greater than one. (See [CK1].) Consequently it follows that $h_{K}^{-}>1$ if $n \geq 49$. For $n \geq 50$ we get (1.1). (In the tables the values $C_{7}(n, b, t)$ and $h(n)$ (resp. $B(n)$ and $\left.c_{6}(2 n, \rho)\right)$ are rounded down (resp. up) after four decimal places.)

Set

$$
N(n, i, b, t)=\frac{1}{\left(1-i^{-2}\right) \exp \left(2 L_{i}(n, b, t)\right)}
$$


TABLE 6. Upper bounds for root discriminants

\begin{tabular}{|c|c|c|c|c|c|c|c|c|}
\hline$n$ & $\rho: \rho \leq \rho_{K}$ & $b$ & $t$ & $c_{4}(n, b, t)$ & $C_{5}(n, b, t)$ & $\alpha(n)$ & $c_{6}(2 n, \alpha(n)) \leq$ & $\operatorname{Bess}(n)$ \\
\hline 48 & 42.479 & 5.105 & 4 & 0.21615 & 37.899 & 50.71 & 6.6778 & 125.2 \\
\hline 40 & 38.491 & 4.921 & 4 & 0.21817 & 36.167 & 54.98 & 6.7432 & 204.0 \\
\hline 32 & 33.770 & 4.721 & 4 & 0.22059 & 33.900 & 61.90 & 6.8245 & 505.6 \\
\hline 30 & 32.444 & 4.669 & 4 & 0.22126 & 33.211 & 64.37 & 6.8482 & 729.3 \\
\hline 24 & 28.017 & 4.494 & 4 & 0.22368 & 30.725 & 75.08 & 6.9303 & 5252 \\
\hline 20 & 24.608 & 4.370 & 4 & 0.22554 & 28.554 & 87.36 & 6.9972 & 6499 \\
\hline 18 & 22.733 & 4.301 & 4 & 0.22664 & 27.250 & 96.55 & 7.0355 & \\
\hline 16 & 20.726 & 4.231 & 4 & 0.22780 & 25.741 & 109.3 & 7.0779 & \\
\hline 15 & 19.668 & 4.195 & 4 & 0.22842 & 24.893 & 117.7 & 7.1008 & 6875 \\
\hline 10 & 13.744 & 3.996 & 4 & 0.23209 & 19.296 & 211.0 & 7.2384 & 7653 \\
\hline 8 & 11.042 & 3.903 & 4 & 0.23397 & 16.106 & 324.7 & 7.3076 & \\
\hline 5 & 6.644 & 3.752 & 4 & 0.23728 & 9.5580 & 1163 & 7.4320 & 10250 \\
\hline
\end{tabular}

if $i$ is a power of a prime, and $N(n, i, b, t)=1$ otherwise. If $N(n, i, b, t) \geq 1$ for $2 \leq i \leq t-1$, then, according to (5.6),

$$
h_{K}^{-} \geq \frac{2}{c_{6}(2 n, \rho)}\left(\frac{C_{5}(n, b, t)^{c_{4}(n, b, t)}}{2 \pi}\right)^{n} \frac{D_{K}^{\frac{1}{4}-\frac{1}{2} c_{4}(n, b, t)}}{\log \log D_{K}} .
$$

In a similar fashion as above, for $5 \leq n \leq 48$ we find optimal values of $b$ and $t$ that minimize $\alpha(n)$ so that $\rho_{K} \geq \alpha(n)$ implies $h_{K}^{-}>1$. We summarize our computational results in Table 6] Here Bess(n) means Bessassi's upper bounds for root discriminants in $[\mathrm{B}$.

6.2. Proof of Theorem 1 point (2). Set $C_{8}(m)=c e^{1 / 2 c} B(m)$ and

$$
P(m, i, b, t)=\frac{\exp \left(\left(1-2 c_{4}(m, b, t)-\frac{2}{m}\right) l_{i}\right)}{\exp \left(2 L_{i}(m, b, t)\right)}
$$

if $i$ is a power of a prime, $P(m, i, b, t)=1$ otherwise. For given $m, b$, and $t$, if $P(m, i, b, t) \geq 1$ for all $i$ with $2 \leq i<t$ and $c_{4}(m, b, t) \leq 0.4$, then, according to (5.4), for every normal CM-field of degree $2 n \geq 2 m \geq 100$ we have

$$
h_{K}^{-} \geq \frac{C_{7}(m, b, t)^{n}}{n C_{8}(m)} \text {. }
$$

In a similar fashion as (1) we get the results in Table 7

The results in Theorem 1 point (2) follow immediately.

6.3. Proof of Theorem 1 point (3). Set $C_{9}(m)=e^{3 / 2}(2 B(m))^{1 / 2} / 2, C_{10}(m)=$ $\exp (1 / \sqrt{2 B(m)})$, and

$$
R(m, i, b, t)=\frac{\exp \left(\left(1-2 c_{4}(m, b, t)\right) l_{i}\right)}{\left(1+\frac{l_{i}}{m B(m)}\right) \exp \left(2 L_{i}(m, b, t)\right)}
$$

if $i$ is a power of a prime, $R(m, i, b, t)=1$ otherwise. For given $m, b$, and $t$, if $c_{4}(m, b, t) \leq 0.4$ and $R(m, i, b, t) \geq 1$ for all $i$ with $i<t$, then, according to (5.5), for any CM-field $K$ of degree $2 n \geq 2 m \geq 10$ we have

$$
h_{K}^{-} \geq \frac{C_{7}(m, b, t)^{n}}{\sqrt{n} C_{9}(m) C_{10}(m)^{1 / \sqrt{n}}} .
$$


TABLE 7 . Lower bounds for $h_{K}^{-}$

\begin{tabular}{|c|c|c|c|c|c|c|}
\hline$n$ & $\rho_{K} \geq$ & $C_{8}(n)$ & $b$ & $t$ & $C_{7}(n, b, t)$ & $h(n): h_{K}^{-} \geq h(n)$ \\
\hline 134 & 44.636 & 6.0568 & 8.866 & 47 & 1.0813 & 43.703 \\
\hline 133 & 44.569 & 6.0544 & 8.866 & 47 & 1.0806 & 37.421 \\
\hline 132 & 44.501 & 6.0520 & 8.774 & 46 & 1.0799 & 32.044 \\
\hline 131 & 44.432 & 6.0495 & 8.678 & 45 & 1.0792 & 27.442 \\
\hline 130 & 44.362 & 6.0470 & 8.678 & 45 & 1.0785 & 23.532 \\
\hline 129 & 44.292 & 6.0445 & 8.678 & 45 & 1.0777 & 20.181 \\
\hline 128 & 44.221 & 6.0419 & 8.678 & 45 & 1.0770 & 17.311 \\
\hline 127 & 44.149 & 6.0394 & 8.678 & 45 & 1.0763 & 14.851 \\
\hline 126 & 44.076 & 6.0367 & 8.678 & 45 & 1.0755 & 12.758 \\
\hline 125 & 44.003 & 6.0341 & 8.678 & 45 & 1.0748 & 10.950 \\
\hline 124 & 43.928 & 6.0314 & 8.678 & 45 & 1.0740 & 9.4016 \\
\hline 123 & 43.853 & 6.0286 & 8.480 & 43 & 1.0732 & 8.0832 \\
\hline 122 & 43.777 & 6.0259 & 8.480 & 43 & 1.0725 & 6.9517 \\
\hline 121 & 43.700 & 6.0230 & 8.480 & 43 & 1.0717 & 5.9807 \\
\hline 120 & 43.622 & 6.0202 & 8.480 & 43 & 1.0709 & 5.1414 \\
\hline 119 & 43.543 & 6.0173 & 8.480 & 43 & 1.0700 & 4.4266 \\
\hline 118 & 43.463 & 6.0144 & 8.480 & 43 & 1.0692 & 3.8127 \\
\hline 117 & 43.382 & 6.0114 & 8.378 & 42 & 1.0684 & 3.2819 \\
\hline 116 & 43.301 & 6.0084 & 8.378 & 42 & 1.0675 & 2.8294 \\
\hline 115 & 43.218 & 6.0054 & 8.378 & 42 & 1.0667 & 2.4380 \\
\hline 114 & 43.134 & 6.0023 & 8.378 & 42 & 1.0658 & 2.1041 \\
\hline 113 & 43.049 & 5.9991 & 8.272 & 41 & 1.0649 & 1.8150 \\
\hline 112 & 42.963 & 5.9960 & 8.272 & 41 & 1.0641 & 1.5666 \\
\hline 111 & 42.876 & 5.9927 & 8.272 & 41 & 1.0632 & 1.3530 \\
\hline 110 & 42.788 & 5.9894 & 8.163 & 40 & 1.0622 & 1.1694 \\
\hline 109 & 42.699 & 5.9861 & 8.163 & 40 & 1.0613 & 1.0103 \\
\hline 108 & 42.609 & 5.9827 & 8.163 & 40 & 1.0604 & 0.8735 \\
\hline & & & & & \\
\hline
\end{tabular}

Similarly as (1) we obtain favorable values for $C_{9}(m), C_{10}(m), C_{7}(m, b, t)$, and lower bounds for $h_{K}^{-}$in Table 8

The results in Theorem 1 point (3) follow immediately.

Remark 3. There is no theoretical reason to take $b$ with $2 \leq b \leq 20$ and $t$ with $t \leq 101$. If we compute $C_{7}(m, b, t)$ with $t>101$ for given $m$ and $b$, we can get better lower bounds for $h_{K}^{-}$. In fact, when $n=83$, varying $b$ between 2 and 20 as in the proof of Theorem 1 we have computed $C_{7}(83, b, t)$ for all $t \geq 3$ until we find $\alpha$ such that $C_{7}(83, b, t)$ is increasing for $3 \leq t \leq \alpha$ and $C_{7}(83, b, \alpha)>C_{7}(83, b, \alpha+1)$. Among all computed $C_{7}(83, b, t)$ 's, $C_{7}(83,18.8,494)=1.18243 \cdots$ is the largest one. We can hence say that for any normal CM-field $K$ of degree $2 n \geq 2 \cdot 83$,

$$
h_{K}^{-} \geq \frac{2}{6.43519} \frac{(1.18243)^{n}}{\log (8.01057 n)} \geq 52473
$$

if we assume the Generalized Riemann Hypothesis.

Remark 4. Our upper bounds for $\rho_{K}$ for a normal CM-field $K$ with $h_{K}^{-}=1$ in Table 6 are sharper than Bessassi's bounds. In the cases of $5 \leq n \leq 23$, Bessassi has used

$$
\kappa_{k} \leq e\left(\frac{e \log D_{k}}{2 n}\right)^{n-1} \quad([\underline{\text { Lou2 }}, \text { Theorem 1] })
$$


TABLE 8.

\begin{tabular}{|c|c|c|c|c|c|c|c|c|}
\hline$n$ & $\rho_{K} \geq$ & $B(n)$ & $C_{9}(n)$ & $C_{10}(n)$ & $b$ & $t$ & $C_{7}(n, b, t)$ & $h(n): h_{K}^{-} \geq h(n)$ \\
\hline 88 & 56.232 & 4.0295 & 6.3614 & 1.4223 & 12.103 & 101 & 1.1940 & 96586 \\
\hline 87 & 55.969 & 4.0248 & 6.3577 & 1.4226 & 12.103 & 101 & 1.1915 & 68066 \\
\hline 86 & 55.703 & 4.0200 & 6.3540 & 1.4229 & 12.103 & 101 & 1.1890 & 48046 \\
\hline 85 & 55.434 & 4.0152 & 6.3502 & 1.4232 & 12.103 & 101 & 1.1865 & 33972 \\
\hline 84 & 55.162 & 4.0103 & 6.3463 & 1.4235 & 12.103 & 101 & 1.1840 & 24048 \\
\hline 83 & 54.887 & 4.0053 & 6.3423 & 1.4238 & 12.103 & 101 & 1.1814 & 17054 \\
\hline 82 & 54.608 & 4.0002 & 6.3383 & 1.4242 & 12.103 & 101 & 1.1788 & 12102 \\
\hline 81 & 54.327 & 3.9950 & 6.3342 & 1.4245 & 12.103 & 101 & 1.1761 & 8605.5 \\
\hline 80 & 54.041 & 3.9898 & 6.3300 & 1.4248 & 12.103 & 101 & 1.1734 & 6123.9 \\
\hline 79 & 53.752 & 3.9844 & 6.3257 & 1.4252 & 12.103 & 101 & 1.1707 & 4367.8 \\
\hline 78 & 53.460 & 3.9789 & 6.3214 & 1.4255 & 12.103 & 101 & 1.1679 & 3118.3 \\
\hline 77 & 53.164 & 3.9734 & 6.3170 & 1.4259 & 12.103 & 101 & 1.1650 & 2230.3 \\
\hline 76 & 52.864 & 3.9677 & 6.3125 & 1.4262 & 12.103 & 101 & 1.1622 & 1597.1 \\
\hline 75 & 52.561 & 3.9620 & 6.3079 & 1.4266 & 12.103 & 101 & 1.1592 & 1145.9 \\
\hline 74 & 52.253 & 3.9561 & 6.3033 & 1.4270 & 12.103 & 101 & 1.1563 & 823.47 \\
\hline 73 & 51.942 & 3.9501 & 6.2985 & 1.4273 & 12.103 & 101 & 1.1533 & 593.03 \\
\hline 72 & 51.626 & 3.9440 & 6.2936 & 1.4277 & 12.103 & 101 & 1.1502 & 427.54 \\
\hline 71 & 51.306 & 3.9378 & 6.2887 & 1.4281 & 12.103 & 101 & 1.1471 & 308.80 \\
\hline 70 & 50.982 & 3.9315 & 6.2836 & 1.4285 & 12.103 & 101 & 1.1439 & 223.47 \\
\hline 69 & 50.654 & 3.9250 & 6.2784 & 1.4290 & 12.103 & 101 & 1.1407 & 161.96 \\
\hline 68 & 50.321 & 3.9184 & 6.2732 & 1.4294 & 12.019 & 99 & 1.1374 & 117.57 \\
\hline 67 & 49.983 & 3.9117 & 6.2678 & 1.4298 & 12.019 & 99 & 1.1340 & 85.559 \\
\hline 66 & 49.640 & 3.9048 & 6.2623 & 1.4303 & 12.019 & 99 & 1.1306 & 62.379 \\
\hline 65 & 49.293 & 3.8978 & 6.2566 & 1.4307 & 11.890 & 96 & 1.1272 & 45.548 \\
\hline 64 & 48.940 & 3.8906 & 6.2508 & 1.4312 & 11.890 & 96 & 1.1237 & 33.353 \\
\hline 63 & 48.582 & 3.8833 & 6.2450 & 1.4317 & 11.757 & 93 & 1.1201 & 24.481 \\
\hline 62 & 48.219 & 3.8758 & 6.2389 & 1.4322 & 10.853 & 75 & 1.1164 & 18.025 \\
\hline 61 & 47.851 & 3.8681 & 6.2327 & 1.4327 & 10.740 & 73 & 1.1127 & 13.300 \\
\hline 60 & 47.477 & 3.8602 & 6.2264 & 1.4332 & 10.740 & 73 & 1.1090 & 9.8425 \\
\hline 59 & 47.097 & 3.8522 & 6.2199 & 1.4338 & 10.440 & 68 & 1.1052 & 7.3018 \\
\hline 58 & 46.711 & 3.8440 & 6.2133 & 1.4343 & 10.440 & 68 & 1.1013 & 5.4316 \\
\hline 57 & 46.318 & 3.8356 & 6.2065 & 1.4349 & 9.839 & 59 & 1.0973 & 4.0520 \\
\hline 56 & 45.920 & 3.8269 & 6.1995 & 1.4355 & 9.839 & 59 & 1.0932 & 3.0321 \\
\hline 55 & 45.515 & 3.8181 & 6.1923 & 1.4361 & 9.693 & 57 & 1.0891 & 2.2753 \\
\hline 54 & 45.103 & 3.8090 & 6.1849 & 1.4367 & 9.383 & 53 & 1.0849 & 1.7115 \\
\hline 53 & 44.685 & 3.7996 & 6.1773 & 1.4373 & 9.218 & 51 & 1.0806 & 1.2923 \\
\hline 52 & 44.259 & 3.7901 & 6.1695 & 1.4380 & 9.218 & 51 & 1.0763 & 0.97862 \\
\hline
\end{tabular}

to obtain upper bounds $\rho_{K}$, however, even though in these cases we obtained better bounds for $\rho_{K}$ using Proposition 5 .

Remark 5. In Theorem 1, points (2) and (3), we cannot obtain lower bounds $\alpha(n)$ for $\rho_{K}$ such that $h_{K}^{-}=1$ would imply $\rho_{K} \leq \alpha(n)$. For $\frac{1}{\exp \left(2 L_{i}(m, b, t)\right)}<1$ we can not remove the term $\prod_{i=2}^{t-1}\left(\frac{1}{\exp \left(2 L_{i}(m, b, t)\right)}\right)^{g_{i}}$ in the inequalities $(5.7)$ through $(5.10)$.

\section{ACKNOWLEDGMENT}

This work was supported by KRF-Grant 2002-041-C00002. 


\section{REFERENCES}

[B] S. Bessassi, Bounds for the degrees of CM-fields of class number one, Acta Arith., 106.3(2003), 213-245. MR1957106 (2003m:11183)

[CK1] K.-Y. Chang and S.-H. Kwon, Class numbers of imaginary abelian number fields, Proc. Amer. Math. Soc.,128(2000), 2517-2528. MR1707511 (2000m:11108)

[CK2] K.-Y. Chang and S.-H. Kwon, The non-abelian normal CM-fields of degree 36 with class number one, Acta Arith., 101(2002), 53-61. MR1879845(2003e:11119)

[CK3] K.-Y. Chang and S.-H. Kwon, The class number one problem for some non-abelian normal CM-fields of degree 48, Math. Comp., 72(2003), 1003-1017. MR1954981 (2003m:11185)

[H] J. Hoffstein, Some analytic bounds for zeta functions and class numbers, Invent. Math., 55(1979), 37-47. MR0553994 (80k:12019)

[HS] M. Hall, Jr. and J. K. Senior, The groups of order $2^{n}(n \leq 6)$, Macmillan, NY, 1964. MR0168631 (29:5889)

[JNO] R. James, M. F. Newman, and E. A. O'Brien, The groups of order 128, J. Algebra, 129(1990), 136-158. MR1037398 (90j:20050)

[Lef] Y. Lefeuvre, Corps diédraux à multiplication complexe principaux, Ann. Inst. Fourier (Grenoble), 50(2000), 67-103. MR1762338(2001g:11166)

[LLO] F. Lemmermeyer, S. Louboutin, and R. Okazaki, The class number one problem for some non-abelian normal CM-fields of degree 24, J. Théor. Nombres Bordeaux, 11(1999), 387406. MR.1745886 (2001j:11104)

[LO1] S. Louboutin and R. Okazaki, Determination of all non-normal quartic CM-fields and of all non-abelian normal octic CM-fields with class number one, Acta Arith., 67.1(1994), 47-92. MR.1292520 (95g:11107)

[LO2] S. Louboutin and R. Okazaki, The class number one problem for some non-abelian normal CM-fields of 2-power degrees, Proceedings London Math. Soc., Vol. 76, Part 3(1998), 523-548. MR 1616805 (99c:11138)

[LOO] S. Louboutin, R. Okazaki, and M. Olivier, The class number one problem for some nonabelian normal CM-fields, Trans. Amer. Math. Soc., 349(1997), 3657-3678. MR1390044 (97k:11149)

[Lou1] S. Louboutin, The class number one problem for the non-abelian normal CM-fields of degree 16, Acta Arith., 82.2(1997), 173-196. MR1477509 (98j:11097)

[Lou2] S. Louboutin, Explicit bounds for residues of Dedekind zeta functions, values of Lfunctions at $s=1$ and relative class numbers, J. Number Theory, 85(2000), 263-282. MR.1802716 (2002i:11111)

[Lou3] S. Louboutin, Explicit lower bounds for residues at $s=1$ of Dedekind zeta functions and relative class numbers of CM-fields, Trans. Amer. Math. Soc., 355(2003), 3079-3098. MR.1974676 (2004f:11134)

[LPCK] S. Louboutin, Y.-H. Park, K.-Y. Chang, and S.-H. Kwon, The class number one problem for the non-abelian normal CM-fields of degree 2pq, Preprint.

[M] G. A. Miller, Determination of all the groups of order 64, Amer. J. Math., 52(1930), 617-634. MR:1507920

[O1] A. M. Odlyzko, Some analytic extimates of class numbers and discriminants, Invent. Math., 29(1975), 275-286. MR0376613(51:12788)

[O2] A. M. Odlyzko, Bounds for discriminants and related estimates for class numbers, regulators and zeros of zeta functions: A Survey of recent results, Sém. Théor. Nombres Bordeaux 2(1990), 119-141. MR1061762 (91i:11154)

[Ob] E. A. O'Brien, The groups of order dividing 256, Bull. Austral. Math. Soc., 39(1989), 159-160.

[P] Y.-H. Park, The class number one problem for the non-abelian normal CM-fields of degree 24 and 40, Acta Arith., 101.1(2002), 63-80. MR1879844 (2002k:11200)

[Poi1] G. Poitou, Minorations de discriminants, Sém. Bourbaki, 28e année (1975/76), 136-153. MR0435033 (55:7995)

[Poi2] G. Poitou, Sur les petits discriminants, Sém. Delange-Pisot-Poitou, 18e année (1976/77), 6-01 - 6-17. MR.0551335 (81i:12007)

[PK] S.-M. Park and S.-H. Kwon, Class number one problem for normal CM-fields, Preprint. 
[PYK] S.-M. Park, H.-S. Yang, and S.-H. Kwon, Class number one problem for the normal CM-fields of degree 32, Preprint.

[S] H.M. Stark, Some effective cases of the Brauer-Siegel theorem, Invent. Math., 23(1974), 135-152. MR0342472 (49:7218)

[TW] A. D. Thomas and G. V. Wood, Group Tables, Shiva Mathematics series 2, 1980. MR.0572793 (81d:20002)

[W] L. C. Washington, Introduction to cyclotomic fields, 2nd ed. (1997), Grad. Texts in Math. 83, Springer-Verlag. MR1421575 (97h:11130)

[Wo] http://mathworld.wolfram.com/Finitegoup.html.

[Y] K. Yamamura, The determination of the imaginary abelian number fields with class number one, Math. Comp., 62(1994), 899-921. MR1218347 (94g:11096)

Department of Mathematics Education, Korea University, 136-701, Seoul, Korea

E-mail address: thisknow@korea.ac.kr

Department of Mathematics Education, Korea University, 136-701, Seoul, Korea

E-mail address: sounhikwon@korea.ac.kr 